\title{
Myocyte Apoptosis During Acute Myocardial Infarction in the Mouse Localizes to Hypoxic Regions but Occurs Independently of p53
}

\author{
Shani Bialik, ${ }^{\ddagger}$ David L. Geenen, ${ }^{\star}$ Isaac E. Sasson, ${ }^{*}$ Rendi Cheng, ${ }^{\star}$ James W. Horner, ${ }^{\ddagger}$ Sydney M. Evans, ${ }^{\S}$ Edith M. Lord, ${ }^{\natural}$ \\ Cameron J. Koch,,$^{\S}$ and Richard N. Kitsis ${ }^{\star \ddagger}$ \\ $*$ Department of Medicine (Cardiology) and ${ }^{\ddagger}$ Department of Cell Biology, Albert Einstein College of Medicine, Bronx, New York 10461; \\ ${ }^{\S}$ Department of Radiation Oncology, University of Pennsylvania School of Medicine, Philadelphia, Pennsylvania 19104; and ${ }^{\mathbb{\$} C a n c e r}$ \\ Center, University of Rochester, Rochester, New York 14627
}

\begin{abstract}
Significant numbers of myocytes die by apoptosis during myocardial infarction. The molecular mechanism of this process, however, remains largely unexplored. To facilitate a molecular genetic analysis, we have developed a model of ischemia-induced cardiac myocyte apoptosis in the mouse. Surgical occlusion of the left coronary artery results in apoptosis, as indicated by the presence of nucleosome ladders and in situ DNA strand breaks. Apoptosis occurs mainly in cardiac myocytes, and is shown for the first time to be limited to hypoxic regions during acute infarction. Since hypoxia-induced apoptosis in other cell types is dependent on p53, and p53 is induced by hypoxia in cardiac myocytes, we investigated the necessity of p53 for myocyte apoptosis during myocardial infarction. Myocyte apoptosis occurs as readily, however, in the hearts of mice nullizygous for $p 53$ as in wild-type littermates. These data demonstrate the existence of a p53-independent pathway that mediates myocyte apoptosis during myocardial infarction. (J. Clin. Invest. 1997. 100:1363-1372.) Key words: programmed cell death $\bullet$ ischemic heart disease $\bullet$ molecular genetics $\bullet$ knockout mice $\bullet$ nitroimidazoles
\end{abstract}

\section{Introduction}

Cell death can occur by necrosis or apoptosis (for review see references 1-3). Necrosis is a passive process for which a distinguishing characteristic is injury to cellular membranes. The two most important consequences of this insult are $(a)$ the breakdown of cellular homeostasis, and $(b)$ the release of intracellular contents, resulting in a marked inflammatory response. In contrast, apoptosis is an active, gene-directed process in which cells initiate their own deaths in response to internal or external stimuli. Apoptosis is characterized by condensation, margination, and degradation of chromatin, cytoskeletal disruption, cell shrinkage, membrane blebbing, and finally, fragmentation of the cell into membrane-enclosed apoptotic bodies. The latter are ingested by phagocytic or

Address correspondence to Richard N. Kitsis, Department of Medicine and Cell Biology, Albert Einstein College of Medicine, 1300 Morris Park Avenue, Bronx, NY 10461. Phone: 718-430-2609; FAX: 718-430-8991; E-mail: kitsis@aecom.yu.edu

Received for publication 16 January 1997 and accepted in revised form 16 June 1997.

J. Clin. Invest.

(C) The American Society for Clinical Investigation, Inc. 0021-9738/97/09/1363/10 \$2.00

Volume 100, Number 6, September 1997, 1363-1372

http://www.jci.org neighboring cells, avoiding an inflammatory response. This mode of death thus serves as an orderly means for multicellular organisms to eliminate unwanted cells without adversely affecting surrounding tissue. For these reasons, apoptosis is a key mechanism by which cell turnover is accomplished during development, homeostasis, and damage surveillance.

Traditionally, cardiac myocyte death during ischemic injury has been thought to occur exclusively by necrosis. Recently, however, this view has been challenged by several studies demonstrating that large numbers of myocytes undergo apoptosis in response to both prolonged ischemia and ischemia followed by reperfusion in rats, rabbits, and humans (4-9, and reviewed in 10). In addition, cultured neonatal cardiac myocytes undergo apoptosis when deprived of oxygen, glucose, and serum, conditions used as an in vitro surrogate for ischemia (11). In fact, hypoxia alone is sufficient to induce apoptosis in primary cultures of neonatal and adult cardiac myocytes $(12,13)$, suggesting that decreased availability of oxygen could be a proximate stimulus for myocyte apoptosis during ischemia. Myocardial ischemia, however, is a complex state characterized by reduced supply of numerous nutrients, inadequate removal of cellular metabolites, and secondary stimuli, such as mechanical stretch, that result from the effects of ischemia on myocyte function. With respect to the latter, cardiac myocyte apoptosis has also been observed in stretched isolated papillary muscles (14) and in models of hemodynamic overload, hypertension, and chronic heart failure (15-18, and reviewed in 10).

One molecular mediator of hypoxia-induced apoptosis is the tumor suppressor p53. p53 is a transcription factor that effects cell cycle arrest or apoptosis in response to a variety of genotoxic and physical stresses (for review see references 3 and 19). Apoptosis may be elicited, in part, by p53-stimulated transcription of the proapoptotic gene bax (20-22), and repression of the antiapoptotic gene $b c l-2(20,23)$. Protein levels of p53 increase in response to DNA damage (19), oxidative stress (24), and significantly, hypoxia (25). p53, in fact, is necessary for hypoxia-induced apoptosis in mouse embryonic fibroblasts and experimentally-derived solid tumors (26). Of significance, p53 is induced by hypoxia in cultured neonatal cardiac myocytes and overexpression of p53 in normoxic myocytes leads to apoptosis (26a). Accordingly, p53 is likely to play a regulatory role in ischemia-related myocyte death as well.

The interest in whether acute myocyte loss during myocardial ischemia occurs by apoptosis is motivated by the dual hypotheses that apoptosis can be inhibited, and that such inhibition will result in reduction in infarct size and improved myocardial function. To address these interrelated issues, however, it is first necessary to delineate the pathways that mediate apoptosis in cardiac myocytes, and to identify key regulatory molecules. To facilitate a molecular genetic approach to this problem, we have developed and characterized a model of ischemia-induced myocyte apoptosis in the mouse. We have 
used this model to assess the relationship between hypoxia and apoptosis in vivo, and to test the necessity of p53 for this process during myocardial infarction (MI). ${ }^{1}$

\section{Methods}

MI model. Myocardial ischemia was induced in the mouse by ligating the left coronary artery, a method previously used in this species (27). 6-8-week-old C57BL/6J male mice (Jackson Laboratories, Bar Harbor, ME) were initially anesthetized with methoxyflurane (PitmanMoore, Mundelein, IL) and ventilated through a nose cone with a tidal volume of $0.2 \mathrm{ml}$ at 120 breaths/min using a rodent respirator (Model 683; Harvard Apparatus, South Natick, MA). Anesthesia was maintained subsequently with $0.5 \%$ isofluorane (Ohmeda PPD, Liberty Corner, $\mathrm{NJ}$ ) delivered in $95 \% \mathrm{O}_{2} / 5 \% \mathrm{CO}_{2}$ through the ventilator. After thoracotomy in the fourth intercostal space, hearts were exteriorized, and the left coronary artery was ligated intramurally $2 \mathrm{~mm}$ from its origin with an 8-0 proline suture. Sham operation involved an identical procedure, except that the suture was passed through the myocardium without tying, and was removed. The chest was closed in two layers, and the animals were allowed to recover. Animals were reanesthetized with methoxyflurane and killed at various time points ranging from $30 \mathrm{~min}$ to $7 \mathrm{~d}$ after coronary ligation. All protocols were approved by the review board of the Animal Institute of the Albert Einstein College of Medicine.

DNA ladder assay. Hearts were removed and rinsed quickly in ice-cold PBS. After removal of the atria and great vessels, heart tissue was frozen in liquid nitrogen, and was stored at $-70^{\circ} \mathrm{C}$ until further analysis. Tissue was ground with a mortar and pestle immersed in liquid nitrogen, and was digested in $1 \mathrm{ml}$ lysis buffer (10 $\mathrm{mM}$ Tris [pH 8.0], $100 \mathrm{mM} \mathrm{NaCl}, 25 \mathrm{mM}$ EDTA, $0.5 \%$ SDS, $1.0 \mathrm{mg} / \mathrm{ml}$ Proteinase $\mathrm{K}$ (Sigma Chemical Co., St. Louis, MO) overnight at $37^{\circ} \mathrm{C}$. Protein was removed by precipitation in $\mathrm{NaCl}$ at a final concentration of $1.2 \mathrm{M}$, followed by centrifugation at $10,000 \mathrm{~g}$ for $30 \mathrm{~min}$. After extraction of supernatants with phenol-chloroform, genomic DNA was precipitated with isopropanol, washed in $70 \%$ ethanol, resuspended in 10 $\mathrm{mM}$ Tris (pH 8.0), $1.0 \mathrm{mM}$ EDTA, and treated with RNase A for 30 $\min$ at $37^{\circ} \mathrm{C}$. Equal quantities of each sample $(30-40 \mu \mathrm{g})$ were subjected to electrophoresis on $1.4 \%$ agarose gels containing $0.5 \mu \mathrm{g} / \mathrm{ml}$ ethidium bromide. Molecular weight marker $(1 \mathrm{~kb})$ was purchased from Gibco BRL (Gaithersburg, MD).

Terminal deoxynucleotidyltransferase (TdT) nick-end labeling (TUNEL)/Evans blue costaining. In a separate cohort of animals that had been subjected to either sham operation or coronary occlusion, the ascending aorta was catheterized through the right carotid artery and retroperfused with ice-cold $0.9 \%$ (wt/vol) saline, followed by $10 \%$ buffered formalin (Sigma Chemical Co.) Approximately 500 $\mu \mathrm{l}$ of Evans blue dye (Sigma Chemical Co.) was then injected retrograde to delineate the region of myocardial perfusion. The heart was excised, sliced into three sections perpendicular to its long axis, and the basal aspect of each section was photographed to record areas stained by Evans blue dye. The slices were then fixed overnight at $4^{\circ} \mathrm{C}$ in $10 \%$ buffered formalin, and were embedded in paraffin. TUNEL was performed on 5- $\mu \mathrm{m}$ sections (TACS 2-TdT Blue Apoptosis Detection Kit; Trevigen Inc., Gaithersburg, MD) according to the manufacturer's suggestions, using a 30-min labeling reaction in the presence of $\mathrm{Co}^{2+}$. Sections were mounted on gridded coverslips (Bellco Glass, Inc., Vineland, NJ) and visualized by light microscopy (Zeiss Axiophot; Carl Zeiss, Inc., Thornwood, NY).

For each section, the number of TUNEL-positive myocyte nuclei in a $0.0625 \mathrm{~mm}^{2}$ area was scored in six anatomically consistent regions

1. Abbreviations used in this paper: EF5, 2-(2-nitro-1H-imidazol-1-yl)$N$-(2,2,3,3,3-pentafluoropropyl) acetamide; LV, left ventricular; MI, myocardial infarction; MLC2v, ventricular myosin light chain 2; TdT, terminal deoxynucleotidyltransferase; TUNEL, TdT nick-end labeling. (see Fig. 4) using a gridded reticule $\times 400$. Apical, midventricular, and basal sections were examined for a total of 18 measurements per heart. Only nuclei that were clearly located in cardiac myocytes were scored. The number of TUNEL-positive myocyte nuclei per $\mathrm{mm}^{2}$ in perfused vs. hypoperfused myocardial regions was determined as follows. Maps of each section were generated using the grids on coverslips as a reference. These were compared with the previously obtained photographs of the Evans blue staining, and each of the 18 regions was classified as perfused or hypoperfused. The average number of TUNEL-positive myocyte nuclei per $\mathrm{mm}^{2}$ in hypoperfused regions was determined by adding the numbers in each hypoperfused region and dividing by the number of such regions $\times 0.0625 \mathrm{~mm}^{2}$. Similar calculations were made for perfused regions. The number of TUNEL-positive myocyte nuclei per $\mathrm{mm}^{2}$ averaged over the whole heart was obtained by summing the numbers of positive nuclei irrespective of perfusion status, and dividing by $18 \times 0.0625 \mathrm{~mm}^{2}$. The total number of myocyte nuclei in the same 18 regions was also counted in serial sections stained with hematoxylin and eosin so that the mean percentage of total myocyte nuclei could be determined in a similar manner. Data at each of five time points is reported as the mean \pm SEM of three hearts. Results were analyzed using the Kruskal-Wallis statistic with differences considered significant at $P<0.05$ (28).

TUNEL/EF5 costaining. To map hypoxic regions of myocardium, $200 \mu \mathrm{l}$ of $10 \mathrm{mM}$ 2-(2-nitro-1H-imidazol-1-yl)- $N$-(2,2,3,3,3-pentafluoropropyl) acetamide (EF5) was administered intravenously 30 min before coronary ligation or sham operation. EF5 forms covalent adducts with intracellular proteins under conditions of low oxygen tension (29). Hypoxic cells can then be identified by immunostaining sections with an antibody against EF5 (30). Hearts were perfused with ice-cold $0.9 \%$ (wt/vol) saline, embedded in OCT (Miles Laboratories, Inc., Elkhart, IN), and frozen by immersion in a slurry of ethanol/dry ice. Frozen sections $(10-\mu \mathrm{m})$ were fixed in $4 \%$ paraformaldehyde for $1 \mathrm{~h}$ at $0^{\circ} \mathrm{C}$, rinsed extensively, and assayed for TUNEL using fluorescein-conjugated dUTP (Apoptag Fluorescent DNA Fragmentation Detection kit; Oncor Inc., Gaithersburg, MD) and immediately photographed. Sections were then blocked and stained with Cy5-conjugated ELK3-51, a monoclonal antibody against EF5, as described (31). For experiments to colocalize hypoxia, apoptosis, and myocytes, sections were simultaneously stained with a rabbit polyclonal antiventricular myosin light chain 2 (MLC2v) antibody (kind gift of Dr. Kenneth Chien, University of California, San Diego), followed by FITC-conjugated goat anti-rabbit secondary antibody (Sigma Chemical Co). Sections were again photographed to document EF5 and/or MLC2v staining, and then counterstained for total nuclei with Hoechst 33342 (Sigma Chemical Co.) and photographed one final time. Photography was performed with a Xillix CCD camera (Xillix Technologies, Vancouver, Canada).

p53 (-/-) mice. Mice heterozygous for a disruption in the $p 53$ gene (kind gift of Drs. Tyler Jacks, Massachusetts Institute of Technology, and Ronald DePinho, Albert Einstein College of Medicine) (32) were bred to produce homozygous knockout and wild-type progeny. Genotyping was performed on tail DNA using a PCR strategy with primers X6.5 (5'-ACAGCGTGGTACCTTAT-3') and X7 (5'TATACTCAGAGCCGGCCT-3') to amplify the endogenous allele, and primers neo18.5 (5'-TCCTCGTGCTTTACGGTATC-3') and $\mathrm{X} 7$ to amplify the disrupted allele. Wild-type and $p 53(-/-)$ mice were then subjected to left coronary artery occlusion or sham operation, and were analyzed by DNA ladder assay or TUNEL as described above.

\section{Results}

Left coronary artery occlusion induces myocyte apoptosis in the intact mouse. Currently, the mouse is the mammal whose genome can be most readily manipulated. Accordingly, we developed and characterized a model of ischemia-induced cardiac myocyte apoptosis in this species so that a genetic 

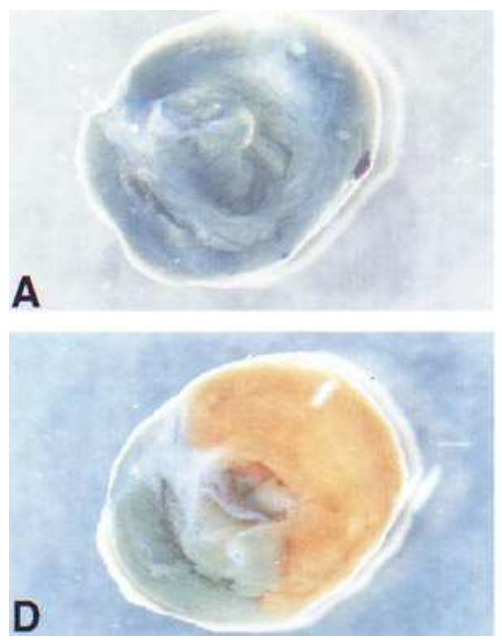
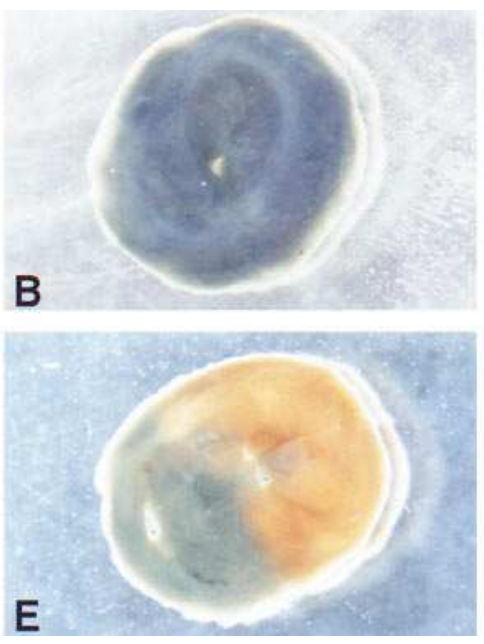
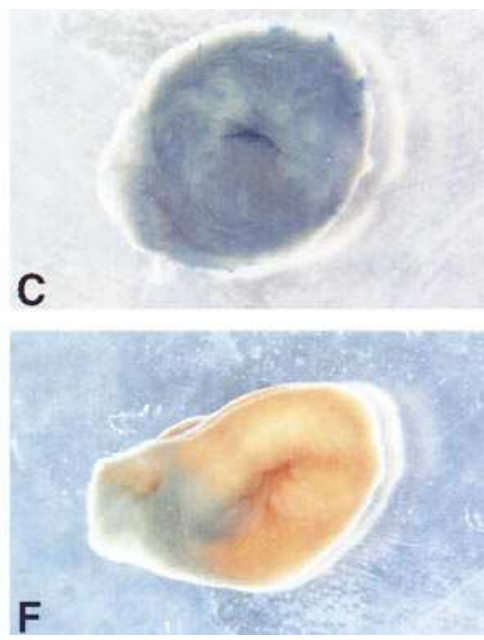

Figure 1. Effect of left coronary artery occlusion on myocardial perfusion. Coomassie Blue dye was injected retrograde into the right carotid artery $30 \mathrm{~min}$ after sham operation or ligation of the left coronary artery. Superior aspects of basal $(A$ and $D)$, midventricular $(B$ and $E)$ and apical $(C$ and $F)$ sections of sham-operated $(A-C)$ and coronary-occluded $(D-F)$ hearts. Perfused regions are accessible to the dye, and therefore appear blue.

approach could be used to investigate the mechanism of this process. Ischemia was produced by ligation of the left coronary artery resulting in a large myocardial perfusion defect that involved predominantly the left ventricular (LV) free wall (Fig. $1, D-F)$. In contrast, perfusion was maintained in sham-operated animals $(A-C)$. Similar results were observed using Evans blue dye after $1,4,10,18$, and $48 \mathrm{~h}$ of ischemia (data not shown).

To determine whether interruption of coronary blood flow stimulates apoptosis in the mouse heart and, if so, to delineate its time course, cardiac DNA was analyzed for nucleosome ladders, a specific marker of apoptosis, at various times after left coronary artery occlusion (Fig. 2). Ladders were com-

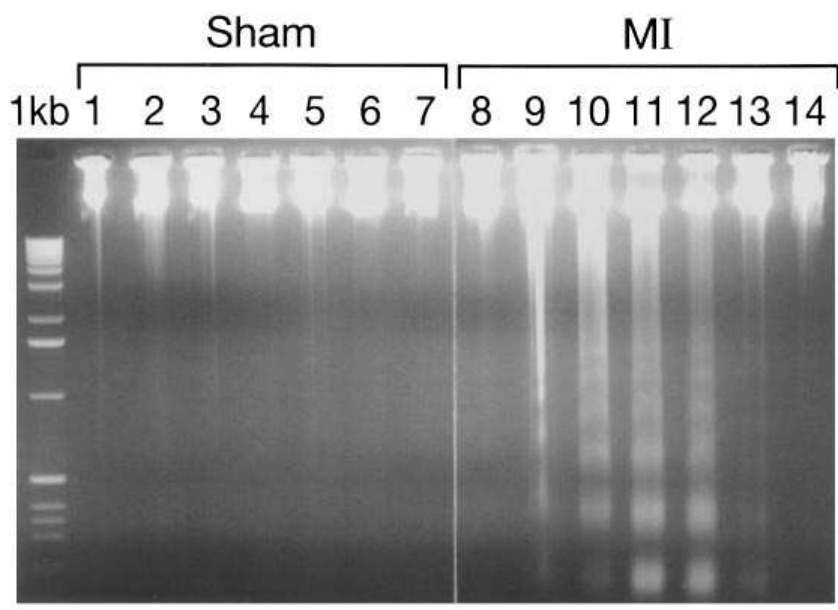

Figure 2. Time course of internucleosomal fragmentation after coronary occlusion. Genomic DNA was isolated from mouse hearts that had been subjected to either sham operation (lanes 1-7) or left coronary artery occlusion (lanes $8-14$ ) and killed $1 \mathrm{~h}$ (lanes 1 and 8 ), $4 \mathrm{~h}$ (lanes 2 and 9), $10 \mathrm{~h}$ (lanes 3 and 10), $18 \mathrm{~h}$ (lanes 4 and 11 ), $48 \mathrm{~h}$ (lanes 5 and 12), $72 \mathrm{~h}$ (lanes 6 and 13) and $7 \mathrm{~d}$ (lanes 7 and 14) later. DNA was resolved on a $1.4 \%$ agarose gel and visualized by ethidium bromide staining. $1 \mathrm{~kb}$ represents DNA molecular weight marker. pletely absent after $1 \mathrm{~h}$ of ischemia (lane 8 ). By $4 \mathrm{~h}$, however, DNA ladders were faintly evident (lane 9) and were clearly present after $10 \mathrm{~h}$ of ischemia (lane 10). The intensity of the ladder pattern increased by $18 \mathrm{~h}$ (lane 11). This remained the case up to $48 \mathrm{~h}$ after left coronary artery ligation (lane 12). By $72 \mathrm{~h}$, however, the intensity of nucleosome ladders was markedly reduced (lane 12), and was completely absent $7 \mathrm{~d}$ after surgery (lane 14). No nucleosome ladders were observed in sham-operated hearts at any of these time points (lanes 1-7). These results were confirmed in a large cohort of mice, which is summarized in Table I. Thus, left coronary artery occlusion induces a time-dependent increase in internucleosomal degradation of cardiac DNA, indicative of apoptosis.

Although the formation of DNA nucleosome ladders is a hallmark of apoptosis, its presence in tissue homogenates provides no information as to the spatial locations or identities of

Table I. Time Course of Apoptosis During MI. Summary of Nucleosome Ladder and TUNEL Assays

\begin{tabular}{|c|c|c|c|c|c|c|}
\hline \multirow[b]{3}{*}{ Time point } & \multirow{2}{*}{\multicolumn{2}{|c|}{$\begin{array}{c}\text { Nucleosome } \\
\text { ladder } \\
\text { present* }\end{array}$}} & \multicolumn{4}{|c|}{ TUNEL-positive myocyte nuclei ${ }^{\ddagger}$} \\
\hline & & & \multicolumn{2}{|r|}{ No. $/ \mathrm{mm}^{2}$} & \multicolumn{2}{|c|}{$\%$ of total myocyte nuclei } \\
\hline & Sham & MI & Sham & MI & Sham & MI \\
\hline $1 \mathrm{~h}$ & $0 / 14$ & $0 / 16$ & 0 & 0 & 0 & 0 \\
\hline $4 \mathrm{~h}$ & $0 / 10$ & $7 / 11$ & 0 & $55.2 \pm 22.7$ & 0 & $3.08 \pm 1.08$ \\
\hline $10 \mathrm{~h}$ & $0 / 11$ & $9 / 10$ & 0 & $117.3 \pm 33.8$ & 0 & $5.21 \pm 1.36$ \\
\hline $18 \mathrm{~h}$ & $0 / 10$ & $16 / 17$ & 0 & $158.8 \pm 113.6$ & 0 & $8.66 \pm 5.17$ \\
\hline $48 \mathrm{~h}$ & $0 / 9$ & $5 / 6$ & 0 & $110.2 \pm 61.4^{\S}$ & 0 & $11.50 \pm 5.93^{\S}$ \\
\hline $72 \mathrm{~h}$ & $0 / 1$ & $4 / 4$ & ND & ND & ND & ND \\
\hline $7 \mathrm{~d}$ & $0 / 2$ & $0 / 4$ & ND & ND & ND & ND \\
\hline
\end{tabular}

$\mathrm{ND}$, not determined. *Expressed as the number of hearts containing nucleosome ladders/total number assayed. ${ }^{*}$ Expressed as mean $\pm \operatorname{SEM}(n=3)$. For each heart, values represent measurements averaged over the entire heart. ${ }^{\S}$ Despite the decrease in the number of positive nuclei $/ \mathrm{mm}^{2}$ between 18 and $48 \mathrm{~h}$, the percentage increases slightly, due to a decrease in the absolute number of remaining myocyte nuclei. 
the particular cell types in which this process is occurring. With respect to the latter, nonmyocytes in the myocardium, including fibroblasts, endothelial cells, and smooth muscle cells, outnumber myocytes by $\sim 4: 1$. To address these issues, TUNEL, an in situ assay of DNA fragmentation, was performed on tissue sections. Fig. 3 shows representative sections from hearts studied $10 \mathrm{~h}(A$ and $B)$ and $18 \mathrm{~h}(C$ and $D)$ after surgery. Consistent with the absence of DNA ladders, no TUNEL-positive nuclei were observed in the hearts of sham-operated animals at either time point ( $A$ and $C$ ). In contrast, both myocyte and nonmyocyte nuclei stained positively for DNA strand breaks upon pretreatment of the section with DNAse I $(F)$. TUNEL- positive nuclei were prevalent in the hearts of animals subjected to left coronary artery occlusion $(B$ and $D)$. This staining did not result from nonspecific binding of reagents to infarcted tissue, as the signal was absent when TdT was omitted from the assay $(E)$. Most, although not all, positively stained nuclei were located within myocytes ( $B$ and $D$ and inset). TUNELpositive myocyte nuclei were most abundant in apical and midventricular sections, and were localized primarily to the LV-free wall. For each heart, the average number of TUNEL-positive myocyte nuclei was obtained from equally spaced measurements made over the entire heart, including both infarcted and noninfarcted myocardium. These values are reported in Table
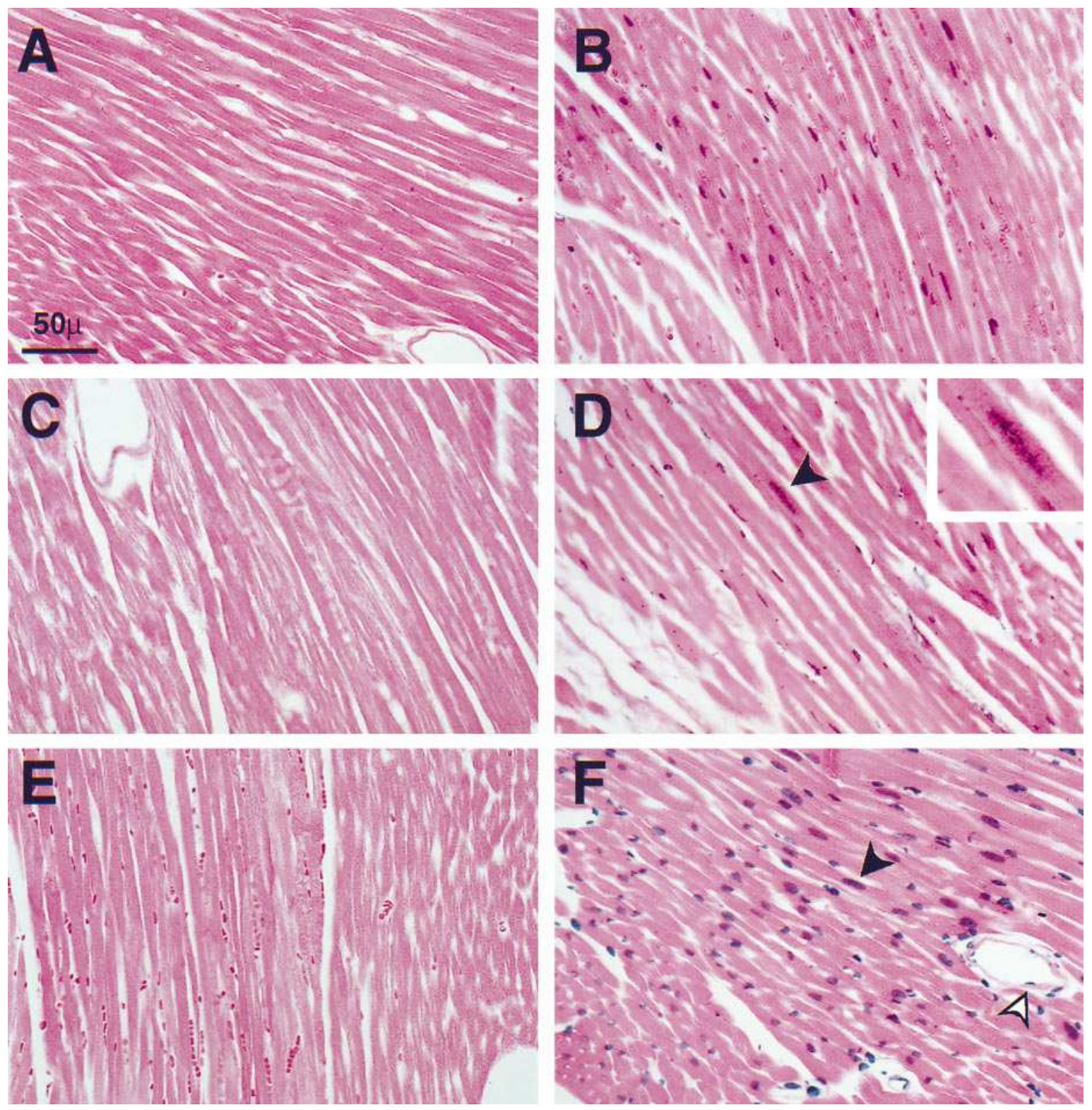

Figure 3. TUNEL staining of heart sections after coronary occlusion. Paraffin-embedded sections of sham-operated $(A$ and $C)$ and infarcted hearts $(B$ and $D)$ at $10(A$ and $B)$ and $18 \mathrm{~h}(C$ and $D)$ were stained for DNA fragmentation by a TUNEL procedure that stains positive nuclei blue. The fields depict the LV free wall at the midventricular level. Positive staining in $B$ and $D$ occurs mainly in myocyte nuclei. Inset of $D$ shows high power magnification of the fragmented myocyte nucleus indicated by the arrow. Shown in $E$ is a serial section of the section in $B$, but incubated in a reaction lacking TdT. $F$ depicts a serial section of the section shown in $A$, pretreated with DNAse I to introduce nonspecific strand breaks as a positive control. Note that both myocyte (closed arrow) and nonmyocyte nuclei (open arrow) stain positive. 
I as the absolute number of positive myocyte nuclei per $\mathrm{mm}^{2}$, and the percentage of total myocyte nuclei that were TUNELpositive. Statistically significant differences were not observed between 4 and $48 \mathrm{~h}$, perhaps due to the variability in the extent of hypoperfusion in our model. Based on the combined DNA ladder and TUNEL data, we conclude that left coronary artery occlusion results in cardiac myocyte apoptosis in the mouse.

Myocyte apoptosis during acute MI occurs primarily in hypoperfused and hypoxic regions. To further investigate the relationship between hypoperfusion and myocyte apoptosis, each heart in the preceding experiment had been retroper- fused with Evans blue dye to delineate regions of hypoperfusion. In this manner, the numbers of TUNEL-positive myocyte nuclei in different sampling areas of known perfusion status were determined. Fig. 4 shows representative data from the hearts of mice subjected to sham operation $(A, C$, and $E$ ) or left coronary artery occlusion $(B, D$, and $F) 18 \mathrm{~h}$ earlier. As stated previously, no TUNEL-positive nuclei were observed in the sham-operated heart. The number of TUNEL-positive myocyte nuclei in each of six $0.0625 \mathrm{~mm}^{2}$ fields are indicated for basal $(B)$, midventricular $(D)$, and apical sections $(F)$ of the infarcted heart. Fig. 5 summarizes the data from 15 animals sub-
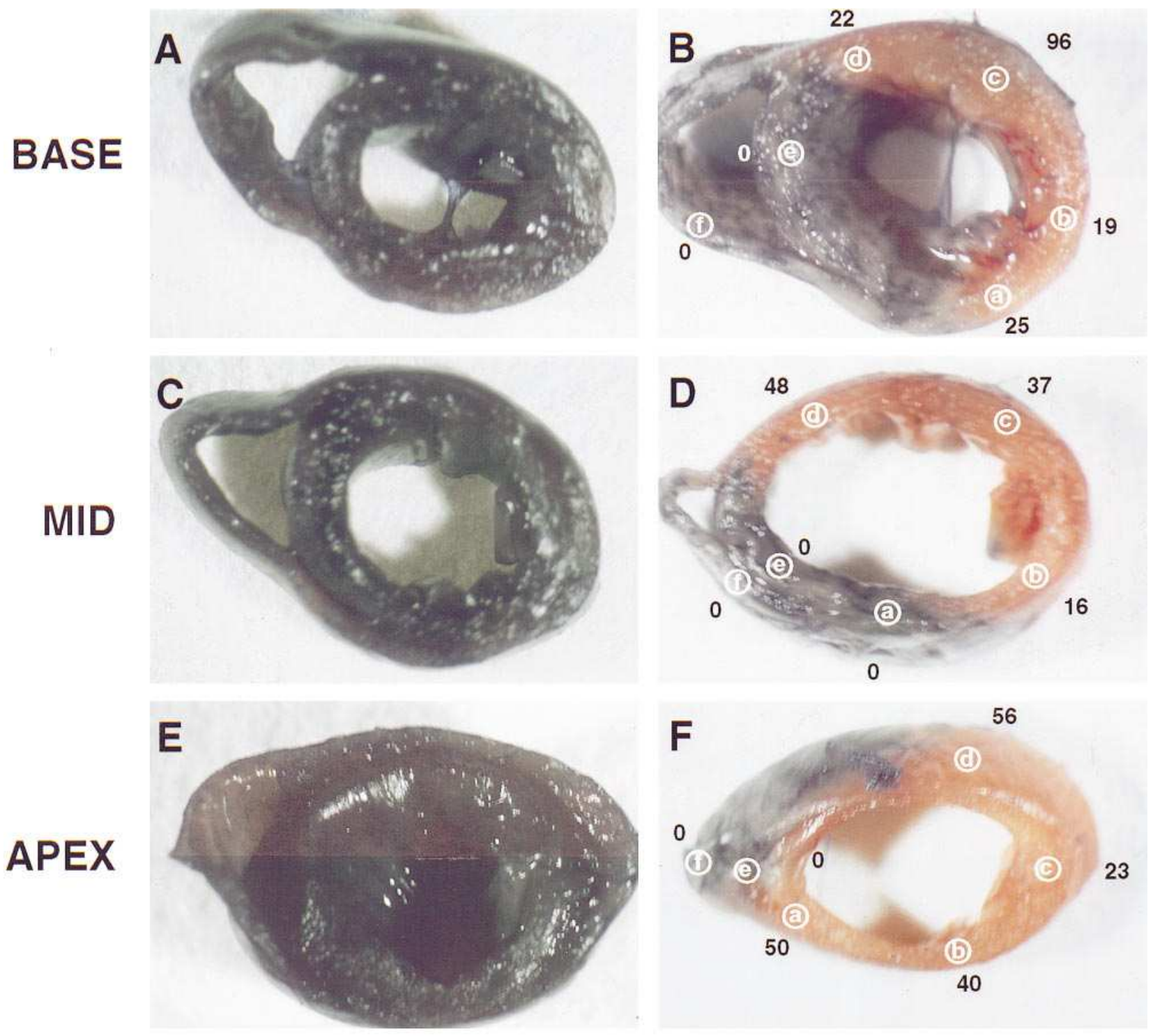

\section{SHAM}

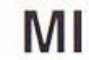

Figure 4. Spatial relationship between hypoperfusion and myocyte apoptosis. $18 \mathrm{~h}$ after sham operation $(A, C$, and $E)$ or coronary occlusion $(B$, $D$, and $F$ ), Evans blue dye was injected as described in the legend to Fig. 1. Basal $(A$ and $B)$, midventricular $(C$ and $D)$ and apical $(E$ and $F)$ sections were photographed to document the extent of perfusion, after which sections were processed for TUNEL staining as in Fig. 3. All regions of the sham-operated heart are perfused, and lack apoptotic cells. For the infarcted heart, the numbers of positive myocyte nuclei were scored in six $0.0625 \mathrm{~mm}^{2}$ fields: four in the LV free wall $(a-d)$, and one each in the septum $(e)$ and right ventricle $(f)$. These quantities are indicated next to their respective areas. Note that TUNEL staining falls within hypoperfused regions. 
jected to left coronary artery occlusion for various time intervals. The number of TUNEL-positive myocyte nuclei per $\mathrm{mm}^{2}$ averaged over the perfused regions in each heart was compared to the number averaged over the hypoperfused regions ( $n=3$ hearts at each time point). In regions of the myocardium that were normally perfused, the average number of TUNEL-positive myocyte nuclei at the $4,10,18$, and $48 \mathrm{~h}$ time points ranged from $0-8 / \mathrm{mm}^{2}$. In contrast, 200-282 such nuclei/ $\mathrm{mm}^{2}$ were seen on average in hypoperfused regions $(P<0.05$ for perfused vs. hypoperfused at each time point). Hypoperfusion did not induce apoptosis uniformly, however, as 10-30\% of hypoperfused regions lacked TUNEL staining. This analysis demonstrates that TUNEL-positive myocyte nuclei are overwhelmingly located in hypoperfused myocardium during acute infarction.

Hypoperfusion deprives the myocardium of many nutrients, including oxygen. A reduction in oxygen tension, or hypoxia, is sufficient to induce apoptosis in cultured cardiac myocytes $(12,13)$. Because of our results showing a strong correlation between hypoperfusion and apoptosis, we assessed whether a similar relationship exists between hypoxia and apoptosis. To demarcate regions of hypoxia, the 2-nitroimidazole compound EF5 was injected intravenously before coronary occlusion. EF5 is reduced under hypoxic conditions, facilitating the formation of covalent linkages with cellular protein thiols $(29,30)$. In contrast, under normal cellular oxygen concentrations, this chemical reaction does not occur, and EF5 diffuses rapidly out of the cell. Since EF5 protein adducts can be identified by immunostaining with a monoclonal antibody against the nitroimidazole $(29,30)$, EF5 provides a marker of hypoxia. This approach has been used successfully to identify hypoxic regions in cell culture and in tissue samples $(26,31)$. As shown

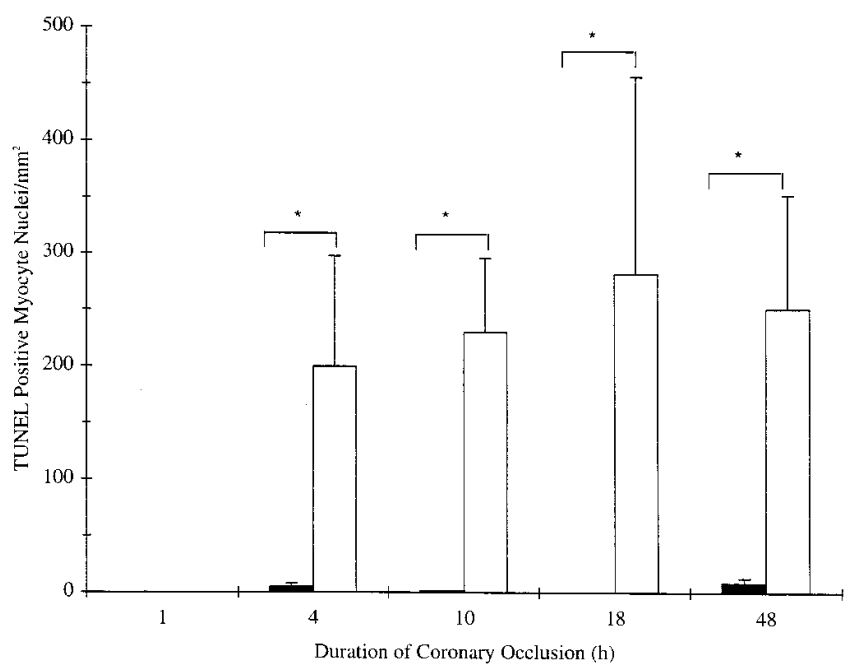

Figure 5. Quantity of TUNEL-positive myocyte nuclei per unit area in perfused and hypoperfused myocardium. At each time point indicated, infarcted hearts were subjected to in situ analysis as described in the legend to Fig. 4. Six fields each in apical, midventricular, and basal cross-sections were scored for each heart. Each field was designated as perfused or hypoperfused based on accessibility to Evans blue dye. The average number of TUNEL-positive myocyte nuclei/ $\mathrm{mm}^{2}$ in perfused (closed bars) or hypoperfused (open bars) fields was calculated for each heart. Data is reported as the mean \pm SEM of three hearts at each time point. *Indicates $P<0.05$. in Figs. $6 B$ and $7 A$, left coronary artery occlusion resulted in EF5 staining in the LV free wall, indicating hypoxia. Since these regions coincided with regions that stained positively for the myocyte marker MLC2v (Fig. 7 C), we conclude that cardiac myocytes were included among the hypoxic cells. This indicates that coronary artery occlusion results in decreased oxygen levels in cardiac myocytes. As expected, the most intense EF5 staining was observed in the subendocardium (Fig. $6 \mathrm{~B}$ ). The cells abutting the LV cavity, which include endocardial cells that are not stained by the myocyte marker (compare $C$ and $D$, Fig. 7), as well as the first few layers of myocytes, were spared from hypoxia, possibly due to direct diffusion of oxygen from the ventricular chamber. Conversely, EF5 staining was absent in a heart subjected to sham operation (Fig. $6 \mathrm{~A}$ ). TUNEL staining of this section was completely negative (data not shown). In infarcted hearts, multiple TUNEL-positive nuclei were evident in EF5-positive regions (Figs. $6 \mathrm{D}$ and $7 \mathrm{~B}$ ). In contrast, TUNEL-positive nuclei were absent from EF5negative regions of these same hearts (Fig. $6 C$ and $7 B$ ). Thus, apoptosis occurred exclusively in hypoxic myocardium. The converse was not true, however; some hypoxic regions lacked apoptotic myocytes (Figs. $6 \mathrm{D}$ and $7 \mathrm{~B}$ ). Taking the Evans blue and EF5 data together, we conclude that cardiac myocyte apoptosis after coronary occlusion correlates strongly with both hypoperfusion and hypoxia.

p53 is not required for myocyte apoptosis during acute MI. The strong correlation between hypoxia and apoptosis after coronary occlusion suggested that oxygen deficiency plays a stimulatory role, as has been demonstrated in vitro $(12,13)$. Furthermore, since p53 is both necessary for hypoxia-induced apoptosis (26) and is sufficient to induce cardiac myocyte apoptosis (26a), we assessed the requirement of p53 for ischemiainduced apoptosis using mice in which the $p 53$ gene had been inactivated by insertional mutagenesis (32). Unexpectedly, nucleosome ladders of similar intensities were evident in both p53 (-/-) and wild-type littermates subjected to left coronary artery occlusion (Fig. 8). This was true at both early (10 h) and late $(48 \mathrm{~h})$ time points for a total of eight knockout mice, indicating that the absence of p53 neither blocked nor delayed myocyte apoptosis. Since it was formally possible that different populations of cells were undergoing apoptosis in the presence and absence of p53, TUNEL staining of heart sections from both genotypes was performed. No differences in the distribution of TUNEL-positive nuclei were observed, however (data not shown). Thus, abrogation of p53 does not affect the occurrence or kinetics of cardiac myocyte apoptosis during MI, implying that apoptosis in this model occurs via a $p 53$-independent pathway.

\section{Discussion}

This study demonstrates that cardiac myocytes undergo apoptosis after coronary occlusion in the mouse. Strong evidence in support of this conclusion is provided by the data from the nucleosome ladder assays, which are specific for apoptosis and the TUNEL assays, which, while not specific for internucleosomal fragmentation, permit identification of the cells that are involved. Apoptotic markers are observed as early as $4 \mathrm{~h}$ after coronary artery ligation, and persist for as long as $48 \mathrm{~h}$. A similar time course was observed in a previous study of cardiac cell death during MI in the rat (7). The persistence of internucleosomal fragmentation suggests that new apoptotic events, in- 

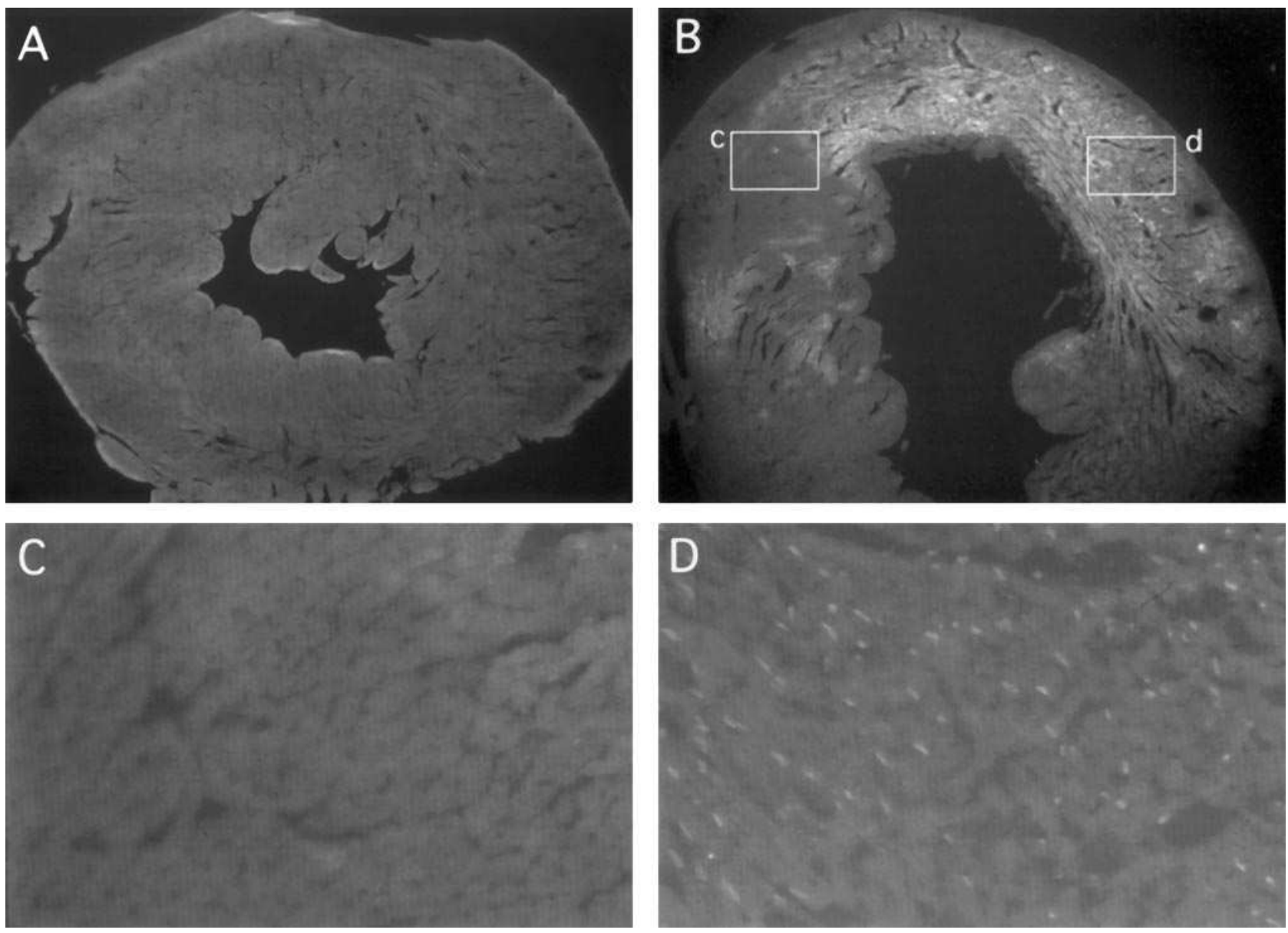

Figure 6. Spatial relationship between hypoxia and apoptosis. Animals were injected with hypoxia marker EF5 30 min before sham operation $(A)$ or left coronary occlusion $(B-D)$. Frozen sections of hearts from mice killed $10 \mathrm{~h}$ after surgery were sequentially stained for TUNEL $(C$ and $D)$ and EF5 ( $A$ and $B, 3 \times 2.5 \mathrm{~mm}^{2}$ field). $C$ and $D$ are high magnification $\left(0.4 \times 0.25 \mathrm{~mm}^{2}\right.$ field $)$ views of TUNEL staining of the boxed regions labeled $c$ and $d$, respectively, in $B$. TUNEL-positive nuclei are found exclusively within regions of EF5 binding.

volving additional myocytes, occur throughout the 48-h period. The basis for this interpretation is the rapidity (minutes to hours) with which apoptosis is executed in some other systems, such as Caenorhabditis elegans $(1,33)$. The kinetics of apoptosis, however, may not be uniform in all cell types and situations (33). For example, in sympathetic neurons deprived of nerve growth factor, more than $12 \mathrm{~h}$ pass from when the first morphological signs of apoptosis are observed until the final stages of cell contraction and membrane blebbing (34). In addition, clearance of apoptotic debris by phagocytosis could be ratelimiting in the heart, resulting in persistence of apoptotic markers. For these reasons, it is possible that one population of myocytes is being detected at multiple time points, a hypothesis that cannot be excluded using currently available apoptosis detection assays. Even the observed temporal increase in the intensity of nucleosomal ladders does not constitute proof of new apoptotic events, as this could reflect further fragmentation of DNA within the same population of cells.

Although there is no doubt that significant necrosis occurs during MI, our estimate that $3-12 \%$ of myocyte nuclei in the entire heart are TUNEL-positive at any given time point suggests that a sizable proportion of myocytes undergo apoptosis. Without knowing the duration of TUNEL positivity after a single apoptotic event, however, we are unable to calculate the cumulative number of apoptotic myocytes over the course of acute infarction. Even so, the minimum rate of myocyte apoptosis as predicted from the above estimate argues that apoptotic cell death is an important contributor to myocyte loss during MI. The potential clinical significance of this observation is that apoptosis, in contrast to necrosis, is an active process that could, in principle, be inhibited. Inhibition of myocyte apoptosis during MI could translate into reduction in infarct size and improvement in cardiac function. While an attractive possibility, however, this outcome is not assured. Prevention of myocyte apoptosis is unlikely to be beneficial if the same cells undergo necrosis a short time later. In addition, even if subsequent necrosis were not a concern, myocytes rescued from apoptosis may not remain functional. Furthermore, removal of damaged myocytes through apoptosis may be a mechanism by which the heart limits the extent of the potentially more destructive process of necrosis with its accompanying inflammation; consequently, inhibition of apoptosis may actually have deleterious effects on cardiac function. Clearly, these questions need to be addressed to evaluate the potential clinical benefit of inhibiting myocyte apoptosis during MI.

The most direct and definitive approach to resolving these 

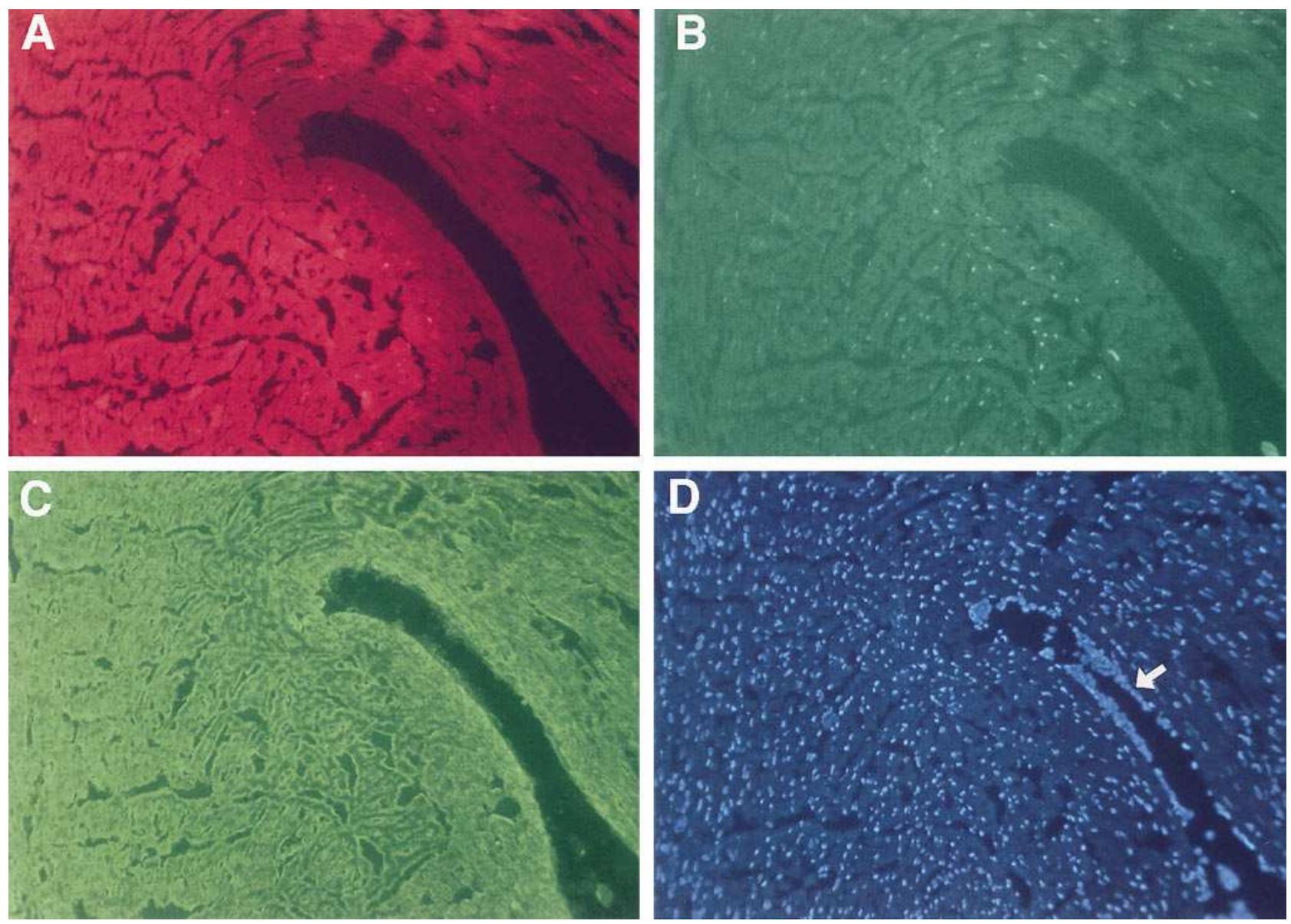

Figure 7. Hypoxia and apoptosis colocalize to cardiac myocytes. After injection of EF5, coronary occlusion was performed, and mice were killed $10 \mathrm{~h}$ later. Frozen sections were sequentially stained and photographed for TUNEL, EF5, and MLC2v, and finally, total nuclei with Hoechst 33342. Shown is the same $0.95 \times 0.70 \mathrm{~mm}^{2}$ field exhibiting EF5 staining ( $A$, Cy5), TUNEL ( $B$, FITC under high gain), MLC2v ( $C$, FITC), and total nuclei $(D)$. TUNEL staining and hypoxia occur within cardiac myocytes. Endocardial cells (indicated with arrow in $D$ ) and myocytes abutting the LV cavity are neither hypoxic nor apoptotic. Note that $B$ was photographed before immunostaining for MLC2v.

issues is to inhibit myocyte apoptosis. To accomplish this, an understanding of the molecular pathways that mediate cardiac myocyte apoptosis is required. The major objectives of the current study were to develop and characterize a mouse model, and to use it to delineate genetic components of ischemiainduced apoptosis. The spatial correlation between hypoperfusion and apoptosis that has been observed here and in a previous study (8) suggests that the death stimulus involves the absence of a substance that would normally be supplied by the circulation. The most obvious nutrient in short supply is oxygen, lack of which may directly lead to cell death by inhibiting oxygen-dependent metabolic processes, as has been demonstrated in vitro in cardiac myocytes $(12,13)$. This hypothesis is supported by the fact that apoptosis during MI was observed only in EF5-positive, hypoxic regions of the myocardium. Not all hypoxic areas were apoptotic, however, and even within those regions that did contain TUNEL-positive nuclei, not all cells were involved. This heterogeneity may be due to subtle differences in the degree of hypoxia within different EF5-positive regions. In fact, the intensity of EF5 staining varied to a certain extent among infarcts, and even within an infarct.

Although the majority of hypoxia-induced apoptosis in tumor cells was inhibited upon disruption of p53 (26), ischemia- induced myocyte apoptosis continues undiminished in its absence. We were not able to evaluate whether there were subtle changes in the quantity of apoptotic cells in the absence of p53, due to inherent variability in the size of perfusion defects in our model. Nevertheless, the degree of apoptosis observed in the p53 knockout mice demonstrates that a major portion of this process must occur via a p53-independent pathway. This is in stark contrast to the low levels of p53-independent apoptosis that were observed in hypoxic tumors (26). This discrepancy may be due to differences in cell type. Alternatively, there may be other p53-independent stimuli that operate in conjunction with hypoxia to elicit myocyte apoptosis after coronary occlusion. These could include deprivation of cardiac survival factors (35) or the secondary consequences of myocardial hypoperfusion, such as increased mechanical load $(9,14$, $15,17)$. In combination with various knockout and transgenic mice, the model described in this report will be useful in identifying alternative stimuli and pathways that mediate myocyte apoptosis. Our results indicate clearly that at least one of these stimuli, whether it be hypoxia or some other factor, activates a p53-independent pathway that leads to the apoptotic cell death of cardiac myocytes.

In conclusion, we describe a mouse model of MI in which 


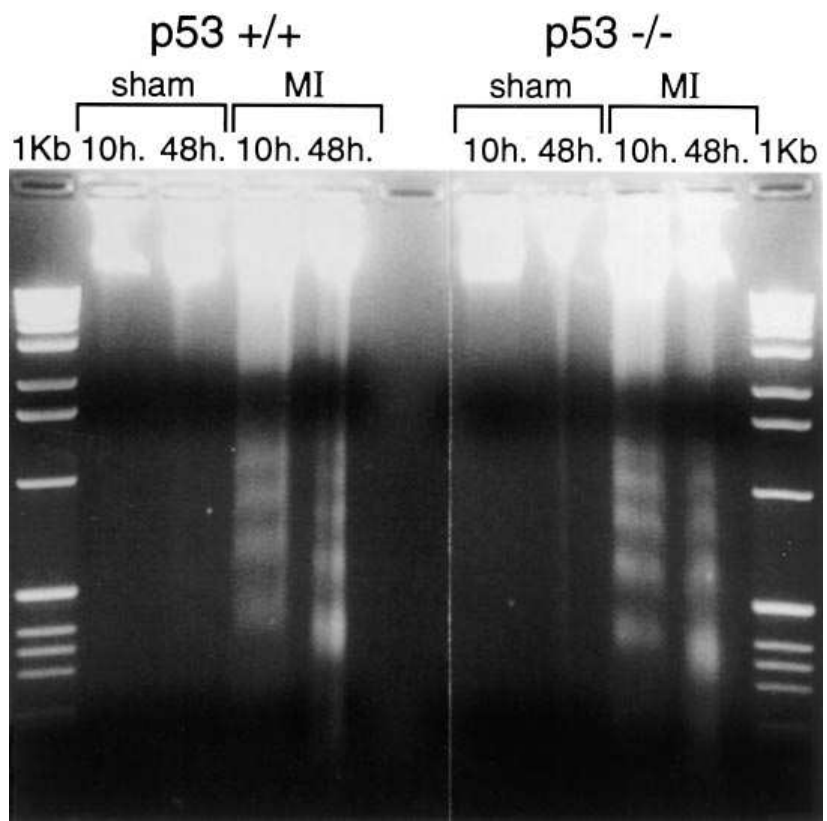

Figure 8. Effect of the absence of $p 53$ on apoptosis during MI. Total cardiac DNA was isolated from wild-type $(p 53+/+)$ or p53 knockout ( $553-1-$ ) mice subjected either to sham operation (sham) or coronary occlusion $(M I)$ and killed after the indicated number of hours. $1 \mathrm{~kb}$ indicates DNA molecular weight marker. See Fig. 2 for experimental details. Apoptosis occurs even in the absence of $p 53$.

cardiac myocyte apoptosis is observed exclusively in hypoxic myocardium over $48 \mathrm{~h}$. This model enables us to delineate the molecular components that regulate this complex process. We have shown, in fact, that myocyte apoptosis following coronary occlusion occurs independently of p53, suggesting that multiple pathways combine to activate apoptosis in the ischemic heart.

\section{Acknowledgments}

The authors wish to acknowledge the technical assistance of Kamala Tamirisa, Aftab Qureshi, Aparna Surendran, and Cecelia Chan, and the generosity of Drs. Tyler Jacks, Ronald DePinho, and Kenneth Chien. We are grateful to Dr. Edmund H. Sonnenblick and Yan Liu for multiple insightful discussions, and to Dr. Stephen Factor for sharing his expertise in cardiac pathology. We thank Dr. Michael Crow for communicating experimental results before publication.

S. Bialik is supported by a Howard Hughes Medical Institute predoctoral fellowship. This work was supported by grants from the NIH to R.N. Kitsis (HL 02699) and C.J. Koch (CA 56679), and D.L. Geenen was supported, in part, by HL 15498. R.N. Kitsis is the Charles and Tamara Krasne Faculty Scholar in Cardiovascular Research of the Albert Einstein College of Medicine.

\section{References}

1. Searle, J., J.F. Kerr, and C.J. Bishop. 1982. Necrosis and apoptosis: distinct modes of cell death with fundamentally different significance. Pathol. Аппи. 17:229-259.

2. Hale, A.J., C.A. Smith, L.C. Sutherland, V.E. Stoneman, V.L. Longthorne, A.C. Culhane, and G.T. Williams. 1996. Apoptosis: molecular regulation of cell death. Eur. J. Biochem. 236:1-26. 15.

3. White, E. 1996. Life, death and the pursuit of apoptosis. Genes Dev. 10:1-
4. Gottlieb, R.A., K.O. Burleson, R.A. Kloner, B.M. Babior, and R.L. Engler. 1994. Reperfusion injury induces apoptosis in rabbit cardiomyocytes. $J$. Clin. Invest. 94:1621-1628.

5. Buerke, M., T. Murohara, C. Skurk, C. Nuss, K. Tomaselli, and A.M. Lefer. 1995. Cardioprotective effect of insulin-like growth factor I in myocardial ischemia followed by reperfusion. Proc. Natl. Acad. Sci. USA. 92:80318035 .

6. Itoh, G., J. Tamura, M. Suzuki, Y. Suzuki, H. Ikeda, M. Koike, M. Nomura, T. Jie, and K. Ito. 1995. DNA fragmentation of human infarcted myocardial cells demonstrated by the nick end labeling method and DNA agarose gel electrophoresis. Am. J. Pathol. 146:1325-1331.

7. Kajstura, J., W. Cheng, K. Reiss, W.A. Clark, E.H. Sonnenblick, S. Krajewski, J.C. Reed, G. Olivetti, and P. Anversa. 1996. Apoptotic and necrotic myocyte cell deaths are independent contributing variables of infarct size in rats. Lab. Invest. 74:86-107.

8. Fliss, H., and D. Gattinger. 1996. Apoptosis in ischemic and reperfused rat myocardium. Circ. Res. 79:949-956.

9. Cheng, W., J. Kajstura, J.A. Nitahara, B. Li, K. Reis, Y. Liu, W.A. Clark, S. Krajewski, J.C. Reed, G. Olivetti, and P. Anversa. 1996. Programmed myocyte cell death affects the viable myocardium after infarction in rats. Exp. Cell Res. 226:316-327.

10. Bialik, S., D.L. Geenen, M.R. Bennett, S. Sivapalasingam, W.H. Frishman, E.H. Sonnenblick, and R.N. Kitsis. 1997. Cardiac myocyte apoptosis: a new therapeutic target? In Cardiovascular Therapeutics. W.H. Frishman and E.H. Sonnenblick, editors. McGraw-Hill, New York. 955-972.

11. Umansky, S.R., G.M. Cuenco, S.S. Khutzian, P.J. Barr, and L.D. Tomei. 1995. Postischemic apoptotic death of rat neonatal cardiomyocytes. Cell Death Diff. 2:235-241.

12. Tanaka, M., I. Hioshi, A. Susumu, A. Hajime, N. Toshio, K. Takeshi, M. Fumiaki, and H. Michiaki. 1994. Hypoxia induces apoptosis with enhanced expression of Fas antigen messenger RNA in cultured neonatal rat cardiomyocytes. Circ. Res. 75:426-433.

13. Rizk, M.M., P.R. Pierzchalski, K. Reiss, J. Kajstura, M.B. Weiss, and P. Anversa. 1996. Hypoxia induces apoptosis and necrosis of adult rat ventricular myocytes in culture. Circulation. 94:I-471. (Abstr.)

14. Cheng, W., B. Li, J. Kajstura, P. Li, M.S. Wolin, E.H. Sonnenblick, T.H. Hintze, G. Olivetti, and P. Anversa. 1995. Stretch-induced programmed myocyte cell death. J. Clin. Invest. 96:2247-2259.

15. Teiger, E., T.-V. Dam, L. Richard, C. Wisnewsky, B.-S. Tea, L. Gaboury, J. Tremblay, K. Schwartz, and P. Hamet. 1996. Apoptosis in pressure overload-induced heart hypertrophy in the rat. J. Clin. Invest. 97:2891-2897.

16. Hamet, P., L. Richard, T.V. Dam, E. Teiger, S.N. Orlov, L. Gaboury, F. Gossard, and J. Tremblay. 1995. Apoptosis in target organs of hypertension. Hypertension (Dallas). 26:642-648.

17. Sharov, V.G., H.N. Sabbah, H. Shimoyama, A.V. Goussev, M. Lesch, and S. Goldstein. 1996. Evidence of cardiocyte apoptosis in myocardium of dogs with chronic heart failure. Am. J. Pathol. 148:141-149.

18. Narula, J., N. Haider, R. Virmani, T.G. DiSalvo, F.D. Kolodgie, R.J. Hajjar, U. Schmidt, M.J. Semigran, G.W. Dec, and B.-A. Khaw. 1996. Apoptosis in myocytes in end stage heart failure. N. Engl. J. Med. 335:1182-1189.

19. Selivanova, G., and K.G. Wiman. 1995. p53: a cell cycle regulator activated by DNA damage. Adv. Cancer Res. 66:143-180.

20. Miyashita, T., S. Krajewski, M. Krajewska, H.G. Wang, H.K. Lin, D.A Liebermann, B. Hoffman, and J.C. Reed. 1994. Tumor suppressor p53 is a regulator of bcl-2 and bax gene expression in vitro and in vivo. Oncogene. 9:17991805 .

21. Selvakumaran, M., H.-K. Lin, T. Miyashita, H.G. Wang, S. Krajewski, J.C. Reed, B. Hoffman, and D. Liebermann. 1994. Immediate early up-regulation of bax expression by 53 but not TGFB1: a paradigm for distinct apoptotic pathways. Oncogene. 9:1791-1798.

22. Miyashita, T., and J.C. Reed. 1995. Tumor suppressor p53 is a direct transcriptional activator of the human bax gene. Cell. 80:293-299.

23. Miyashita, T., M. Harigai, M. Hanada, and J.C. Reed. 1994. Identification of a p53-dependent negative response element in the bcl-2 gene. Cancer Res. 54:3131-3135.

24. Schwartz, J.L., D.Z. Antoniades, and S. Zhao. 1993. Molecular and biochemical reprogramming of oncogenesis through the activity of prooxidants and antioxidants. Ann. NY Acad. Sci. 686:262-278.

25. Graeber, T.G., J.F. Peterson, M. Tsai, K. Monica, A.J. Fornace, Jr., and A.J. Giaccia. 1994. Hypoxia induces accumulation of $\mathrm{p} 53$ protein, but activation of a G1-phase checkpoint by low-oxygen conditions is independent of p53 status. Mol. Cell. Biol. 14:6264-6277.

26. Graeber, T.G., C. Osmanian, T. Jacks, D.E. Housman, C.J. Koch, S.W. Lowe, and A.J. Giaccia. 1996. Hypoxia-mediated selection of cells with diminished apoptotic potential in tumours. Nature (Lond.). 379:88-91.

26a. Long, X., M.O. Boluyt, M. dL. Hipolito, M.S. Lundberg, J.-S. Zheng, L. O'Neil, C. Cirielli, E.G. Lakatta, and M.T. Crow. 1997. p53 and the hypoxia-induced apoptosis of cultured neonatal rat cardiac myocytes. J. Clin. Invest. 99:2635-2643.

27. Michael, L.H., M.L. Entman, C.J. Hartley, K.A. Youker, J. Zhu, S.R. Hall, H.K. Hawkins, K. Berens, and C.M. Ballantyne. 1995. Myocardial ischemia and reperfusion: a murine model. Am. J. Physiol. 269:H2147-H2154. 
28. Glantz, S.A. 1992. Primer of Biostatistics. 3rd ed. McGraw-Hill Inc., New York.

29. Lord, E.M., L. Harwell, and C.J. Koch. 1993. Detection of hypoxic cells by monoclonal antibody recognizing 2-nitroimidazole adducts. Cancer Res. 53: 5721-5726.

30. Koch, C.J., S.M. Evans, and E.M. Lord. 1995. Oxygen dependence of cellular uptake of EF5 (2-[2-nitro-1H-imidazole-1-yl]- $N$-(2,2,3,3,3-pentafluoropropyl)acetamide): analysis of drug adducts by fluorescent antibodies vs. bound radioactivity. Brit. J. Cancer. 72:865-870.

31. Evans, S.M., C.J. Koch, B. Joiner, W.T. Jenkins, K.M. Laughlin, and E.M. Lord. 1995. 2-nitroimidazole binding for identification of hypoxic cell fraction in cells and tissues of epigastric 9L tumors. Brit. J. Cancer. 72:875-882.
32. Lowe, S.W., E.M. Schmitt, S.W. Smith, B.A. Osborne, and T. Jacks. 1993. p53 is required for radiation-induced apoptosis in mouse thymocytes. $\mathrm{Na}$ ture (Lond.). 362:847-849.

33. Ellis, R.E., J.Y. Yuan, and H.R. Horvitz. 1991. Mechanisms and functions of cell death. Annu. Rev. Cell Biol. 7:663-698

34. Martin, D.P., R.E. Schmidt, P.S. DiStefano, O.H. Lowry, J.G. Carter, and E.M. Johnson, Jr. 1988. Inhibitors of protein synthesis and RNA synthesis prevent neronal cell death caused by nerve growth factor deprivation. J. Cell Biol. 106:829-843.

35. Sheng, Z., D. Pennica, W.I. Wood, and K.R. Chien. 1996. Cardiotrophin-1 displays early expression in the murine heart tube and promotes cardiac myocyte survival. Development (Cambridge). 122:419-428. 